\title{
On the occurrence of Berezinskii-Kosterlitz-Thouless behavior in highly anisotropic cuprate superconductors
}

\author{
T. Schneider \\ Physikinstitut, University of Zurich, Winterthurerstrasse 190, 8057 Zurich, Switzerland.
}

(Dated: April 9, 2018)

\begin{abstract}
The conflicting observations in the highly anisotropic $\mathrm{Bi}_{2} \mathrm{Sr}_{2} \mathrm{CaCu}_{2} \mathrm{O}_{8+\delta}$, evidence for BKT behavior emerging from magnetization data and smeared 3D-xy behavior, stemming form the temperature dependence of the magnetic in-plane penetration depth are traced back to the rather small ratio, $\xi_{c}^{+} / \xi_{c}^{-}=\xi_{c 0}^{+} / \xi_{c 0}^{-} \simeq 0.45$, between the $c$-axis correlation length probed above $\left(\xi_{c}^{+}\right)$and below $\left(\xi_{c}^{-}\right) T_{c}$ and the comparatively large anisotropy. The latter leads to critical amplitudes $\xi_{c 0}^{ \pm}$which are substantially smaller than the distance between two $\mathrm{CuO}_{2}$ double layers. In combination with $\xi_{c}^{+} / \xi_{c}^{-} \simeq 0.45$ and in contrast to the situation below $T_{c}$ the c-axis correlation length $\xi_{c}^{+}$exceeds the distance between two $\mathrm{CuO}_{2}$ double layers very close to $T_{c}$ only. Below this narrow temperature regime where $3 \mathrm{D}$-xy fluctuations dominate, there is then an extended temperature regime where the units with two $\mathrm{CuO}_{2}$ double layers are nearly uncoupled so that $2 \mathrm{D}$ thermal fluctuations dominate and BKT features are observable.

PACS numbers: 74.72.-h, 74.25.Ha, 64.60.Fr
\end{abstract}

Since the pioneering work of Berezinskii [1], Kosterlitz and Thouless 2] (BKT) on the BKT transition in the two-dimensional (2D) XY model, much efforts have been devoted to observe the universal behavior characteristic of the KT transition, as the universal jump of the superfluid density [3], measured in ${ }^{4} \mathrm{He}$ superfluid films, or the non-linear I - V characteristic, observed in thin films of conventional superconductors [4, 5]. Signatures of BKT physics can be expected also in layered superconductors with weak inter-layer coupling. Potential candidates are underdoped cuprate superconductors where the anisotropy increases with reduced transition temperature $T_{c}[\underline{6}]$. Recent studies of the I-V characteristic [7], the frequency dependent conductivity 8], the Nernst signal[9], the magnetization 10, 11, and of the resistance 12 have been interpreted as signatures of BKT behavior. On the other hand, several experiments $[13,14,15,16,17$, 18, 19, 20, 21] failed to observe any trace of the universal jump in the superfluid density around $T_{c}$. Indeed, a systematic finite-size scaling analysis of in-plane penetration depth data taken on films and single crystals of the highly anisotropic $\mathrm{Bi}_{2} \mathrm{Sr}_{2} \mathrm{CaCu}_{2} \mathrm{O}_{8+\delta}$ reveal a smeared transition, consistent with an inhomogeneity induced finite-size effect 22]. Furthermore, there is evidence that in small thin-film samples on insulating substrates, edge effects modify the vortex-vortex interaction making it short-range, unlike the logarithmic long-range interaction needed for the BKT transition. This appears to make the BKT transition impossible in thin films of any size if they are supported by a non-superconducting substrate 23].

In this work we attempt to unravel the conflicting observations on the highly anisotropic $\mathrm{Bi}_{2} \mathrm{Sr}_{2} \mathrm{CaCu}_{2} \mathrm{O}_{8+\delta}$ : evidence for BKT behavior emerging from the magnetization data of Li et al. [10] and evidence for smeared 3D-xy behavior, stemming form the temperature dependence of the magnetic in-plane penetration depth [13, 16, 22]. Al- though $\mathrm{Bi}_{2} \mathrm{Sr}_{2} \mathrm{CaCu}_{2} \mathrm{O}_{8+\delta}$ is highly anisotropic [24], resistivity measurements [25] uncover clearly the three dimensional nature of the transition in this extreme type II superconductor. Accordingly, sufficiently close to $T_{c}$ homogeneous samples are expected to exhibit 3D-xy critical behavior. To explore how close this should be, we consider the magnetic in-plane penetration data shown in Fig. 1] derived from the complex conductivity measurements of Osborn et al. [16] on epitaxially grown $\mathrm{Bi}_{2} \mathrm{Sr}_{2} \mathrm{CaCu}_{2} \mathrm{O}_{8+\delta}$ films, using a two-coil inductive technique at zero applied. For comparison we included the leading 3D-xy behavior given by the universal relation [5, 22, 26, 27]

$$
\frac{1}{\lambda_{a b}^{2}(T)}=\frac{16 \pi^{3} k_{B} T}{\Phi_{0}^{2} \xi_{c}^{-}(T)}, \xi_{c}^{-}=\xi_{c 0}^{-}|t|^{-\nu}
$$

with

$$
t=T / T_{c}-1, \nu \simeq 2 / 3 .
$$

Apparently, there is a rounded transition pointing to a finite size effect, preventing the $c$-axis correlation length $\xi_{c}^{-}$to grow beyond the limiting length $L_{c}$, set by inhomogeneities. Indeed the extreme in $d \operatorname{Re}(\rho) / d T$ exhibits an inflection point at $T=T_{p_{c}} \simeq 83.77 \mathrm{~K}$ where $\xi_{c}^{-}$attains the limiting length $L_{c}$. As shown previously [22], Eq. (11) yields together with $T_{c}=84 \mathrm{~K}, T_{p_{c}} \simeq 83.77 \mathrm{~K}$, $\operatorname{Re}\left(\rho\left(T_{c}\right)\right) / \operatorname{Re}(\rho(T=0))=0.011, \operatorname{Re}(\rho(T=0))=0.6$ $\mathrm{K}, \operatorname{Re}\left(\rho\left(T_{p_{c}}\right)\right)=13.66 \mathrm{~K}, \lambda_{a b}(0)=265 \mathrm{~nm}$, and $L_{c}=$ $\xi_{c}^{-}\left(T_{p_{c}}\right)$ the estimates

$$
\xi_{c 0}^{-} \simeq 2 \AA, L_{c} \simeq 93 \AA \text {, }
$$

for the critical amplitude of the $c$ - axis correlation length below $T_{c}$ and the limiting length $L_{c}$ set by inhomogeneities. The rather small value of $\xi_{c 0}^{-}$, reflecting the 


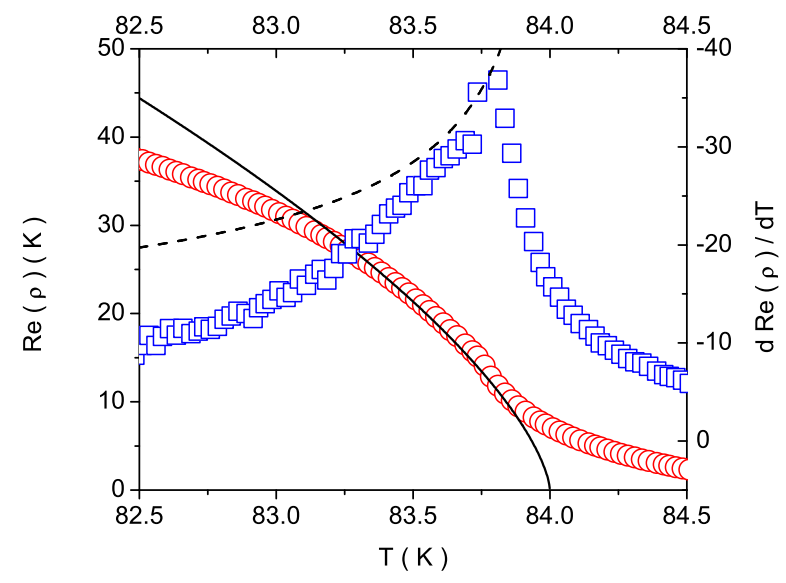

FIG. 1: $\operatorname{Re}(\rho) \propto 1 / \lambda_{a b}^{2}$ and $d \operatorname{Re}(\rho) / d T$ vs. $T$ for the $\mathrm{Bi}_{2} \mathrm{Sr}_{2} \mathrm{CaCu}_{2} \mathrm{O}_{8+\delta}$ film B, $616 \AA$ thick, derived from Osborn et al. [16] The solid and dashed lines indicate the leading critical behavior of a homogeneous bulk system according to Eq. 1 where $\operatorname{Re}(\rho)=650|t|^{2 / 3}$ with $T_{c}=84 \mathrm{~K}$.

high anisotropy, $\gamma=\xi_{a b 0}^{-} / \xi_{c 0}^{-}$, was confirmed in other $\mathrm{Bi}_{2} \mathrm{Sr}_{2} \mathrm{CaCu}_{2} \mathrm{O}_{8+\delta}$ films and single crystals in terms of a detailed finite size scaling analysis [22].

Although the $c$-axis correlation length $\xi_{c}^{-}$increases by approaching $T_{c}$, the occurrence of $3 \mathrm{D}$ thermal fluctuations requires that in $\mathrm{Bi}_{2} \mathrm{Sr}_{2} \mathrm{CaCu}_{2} \mathrm{O}_{8+\delta}$ it exceeds $s=15$ $\AA$, the distance between two $\mathrm{CuO}_{2}$ double layers. In Fig. 2 we depicted the temperature dependence of $\xi_{c}^{-}$. Even though it exceeds $15 \AA$ around $80 \mathrm{~K}$, a glance to Fig. 1 shows that consistency with 3D-xy behavior is achieved much closer to $T_{c}$ only, namely above $T \approx 83.25 \mathrm{~K}$. Accordingly, the occurrence of $3 \mathrm{D}$-xy behavior requires $\xi_{c}^{-}$ to exceed the distance between two $\mathrm{CuO}_{2}$ double layers by at least a factor of three. Above $T_{c}$ the situation is even worse because the ratio between the correlation lengths above $\left(\xi_{c}^{+}\right)$and below $\left(\xi_{c}^{-}\right) T_{c}$ is a universal quantity and in the $3 \mathrm{D}$-xy universality class given by

$$
\xi_{c 0}^{+} / \xi_{c 0}^{-} \simeq 0.45 .
$$

This ratio implies a substantial shrinkage of the 3D-xy fluctuation dominated regime above $T_{c}$. To illustrate this point we included in Fig. 2 the temperature dependence of $\xi_{c}^{+}$. Taking again $\xi_{c}^{+} \gtrsim 3 s=45 \AA$ to locate the regime where 3D-xy fluctuations dominate we obtain roughly $T \gtrsim 83.8 \mathrm{~K}$ which is rather close to the $T_{c} \simeq 84 \mathrm{~K}$ of the fictitious homogeneous system.

Given the reduced 3D-xy critical regime above $T_{c}$ the system corresponds to a stack of nearly independent units with two $\mathrm{CuO}_{2}$ double layers as long as $\xi_{c}^{+} \lesssim 3 s$. In this intermediate regime, $2 \mathrm{D}$ and in particular BKT features are then expected. To check this expectation quantitatively we consider the $3 \mathrm{D}$-xy scaling expression for the susceptibility in the limit $T \gtrsim T_{c}$ and $H_{c} \rightarrow 0$

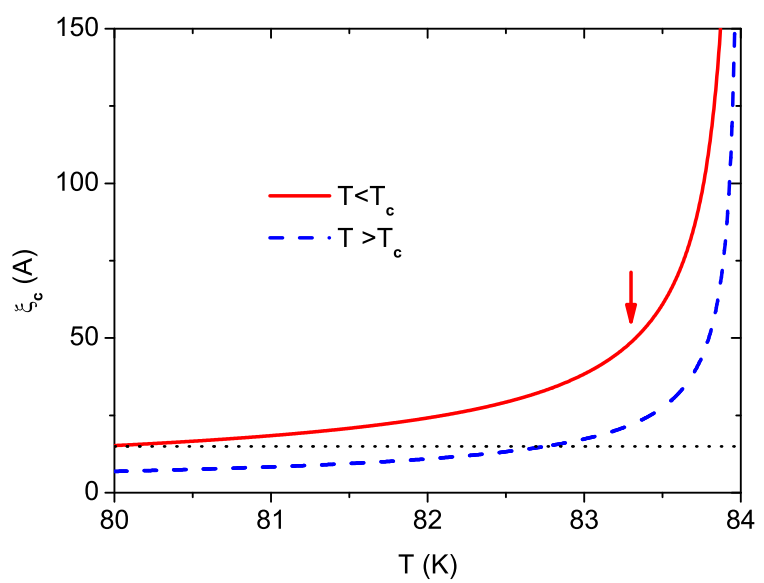

FIG. 2: $\xi_{c}^{-}$and $\xi_{c}^{+}$vs. $T$ for $\xi_{c 0}^{-}=2 \AA, \xi_{c 0}^{+}=0.9 \AA$ and $T_{c}=84 \mathrm{~K}$, where $\xi_{c}^{ \pm}=\xi_{c 0}^{ \pm}|t|^{-2 / 3}$. The dotted line marks $s=15 \AA$, the distance between two $\mathrm{CuO}_{2}$ double layers. The arrow marks $T=83.25 \mathrm{~K}$ where in Fig. 1 consistency with $3 \mathrm{D}-\mathrm{xy}$ critical behavior sets in.

[5, 28, 29, 30]

$$
\frac{m}{H_{c}}=-\frac{Q^{+} C_{3,0}^{+} k_{B} T\left(\xi_{a b}^{+}\right)^{2}}{\Phi_{0}^{2} \xi_{c}^{+}},
$$

where $m=M / V$ is the magnetization per unit volume and $Q^{+} C_{3,0}^{+} \simeq 0.9$ a universal number. In type II superconductors, exposed to a magnetic field $H_{i}$ in direction $i$, there is also the magnetic field induced limiting length $L_{H_{i}}=\sqrt{\Phi_{0} /\left(a H_{i}\right)}$ with $a \simeq 3.12$ [31], related to the average distance between vortex lines [26, 31]. As the magnetic field increases, the density of vortex lines becomes greater, but this cannot continue indefinitely, the limit is roughly set on the proximity of vortex lines by the overlapping of their cores. Due to these limiting length the phase transition is rounded and occurs smoothly. Indeed, approaching $T_{c}$ from above the correlation lengths combination $\xi_{i}^{+} \xi_{j}^{+}$increases but is bounded by $L_{H_{k}}^{2}=\Phi_{0} /\left(a H_{k}\right)$ where $i \neq j \neq k$. In this context it is important to recognize that the confinement effect of the magnetic field in direction i on fluctuations within a region $L_{H_{i}}$ acts only in the plane perpendicular to the field. Therewith Eq. (5) reduces for $T \simeq T_{c}$ and $H_{c} \rightarrow 0$ to

$$
\frac{m}{H_{c}}=-\frac{Q^{+} C_{3,0}^{+} k_{B} T \gamma}{\Phi_{0}^{3 / 2} a^{1 / 2} H_{c}^{1 / 2}},
$$

because $\xi_{a b}^{+} / \xi_{c}^{+}=\gamma$ and the growth of $\xi_{a b}^{+}$is limited by $\xi_{a b}^{+}=L_{H_{c}} \simeq \sqrt{\Phi_{0} /\left(a H_{c}\right)}$. On the other hand, in the strict $2 \mathrm{D}$ case $\xi_{c}^{+}$cannot grow beyond the thickness $d_{s}$ of the system. Consequently, as $-m / H_{c}$ and $\xi_{a b}^{+}$initially increase with reduced temperature in a fixed field they 


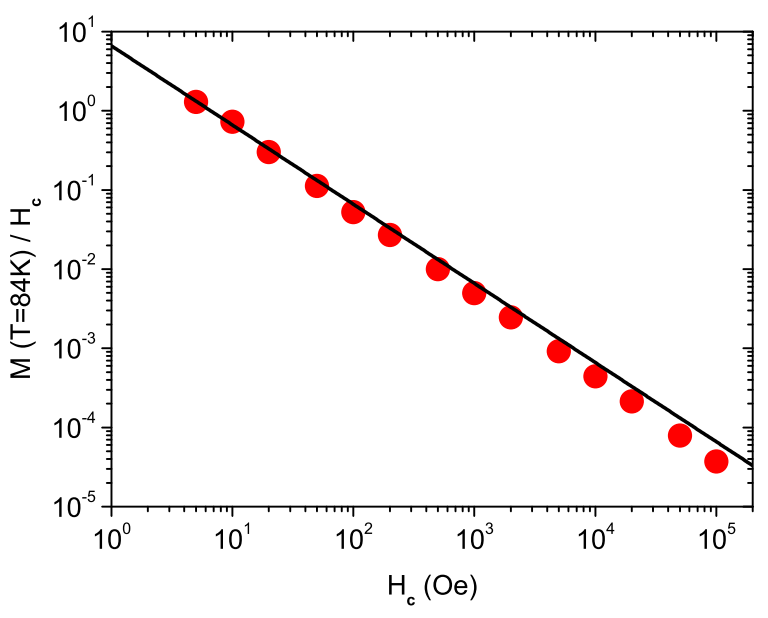

FIG. 3: $M / H$ vs. $H$ for $\mathrm{Bi}_{2} \mathrm{Sr}_{2} \mathrm{CaCu}_{2} \mathrm{O}_{8+\delta}$ at $T=84 \mathrm{~K}$ derived from the magnetization data of Li et al. [10] with $H$ applied along the $c$-axis. The solid line is $M(T=84 K) / H=$ 6.6/H corresponding to Eq. (77).

then saturate due to the magnetic field induced finite size effect. In this case Eq. (5) reduces to

$$
\frac{m}{H_{c}}=-\frac{Q^{+} C_{3,0}^{+} k_{B} T}{\Phi_{0} d_{s} a H_{c}} .
$$

In Fig. 3 we show $-M / H$ vs. $H$ for $\mathrm{Bi}_{2} \mathrm{Sr}_{2} \mathrm{CaCu}_{2} \mathrm{O}_{8+\delta}$ close to $T_{c}$ at $T=84 \mathrm{~K}$ derived from the magnetization data of $\mathrm{Li}$ et al. [10], with $H$ applied along the $c$-axis. The comparison with the characteristic 2D-behavior (7) reveals remarkable agreement, extending over nearly two decades of the applied magnetic field. Noting that down to the lowest applied magnetic field, $H_{c}=5$ Oe, there is no sign of leveling off, arising from an inhomogeneity induced finite size effect, it follows that the length of the homogenous regions in the $a b$-plane, $L_{a b}$, exceeds the attained magnetic field induced limiting length $L_{H_{c}}=\sqrt{\Phi_{0} /\left(a H_{c}\right)}=1.1410^{-4} \mathrm{~cm}$. This behavior does not confirm the occurrence of intermediate $2 \mathrm{D}$ critical behavior in sufficiently anisotropic systems above $T_{c}$ only. In addition, it uncovers that in the $a b$-plane excellent homogeneity can be achieved and an applied magnetic field leads to the outlined finite size effect.

To substantiate the occurrence of the magnetic field induced finite size effect further, we consider the temperature dependence of the magnetization at fixed magnetic field. When $\xi_{c}^{+}<3 s$ and $L_{H_{c}}>>\xi_{a b}^{+}$Eq. (5) reduces to

$$
\frac{m}{T H_{c}}=-\frac{Q^{+} C_{3,0}^{+} k_{B}\left(\xi_{a b}^{+}\right)^{2}}{\Phi_{0}^{2} d_{s}} .
$$

In this regime the in-plane correlation length $\xi_{a b}^{+}$is expected to exhibit the characteristic BKT-behavior in

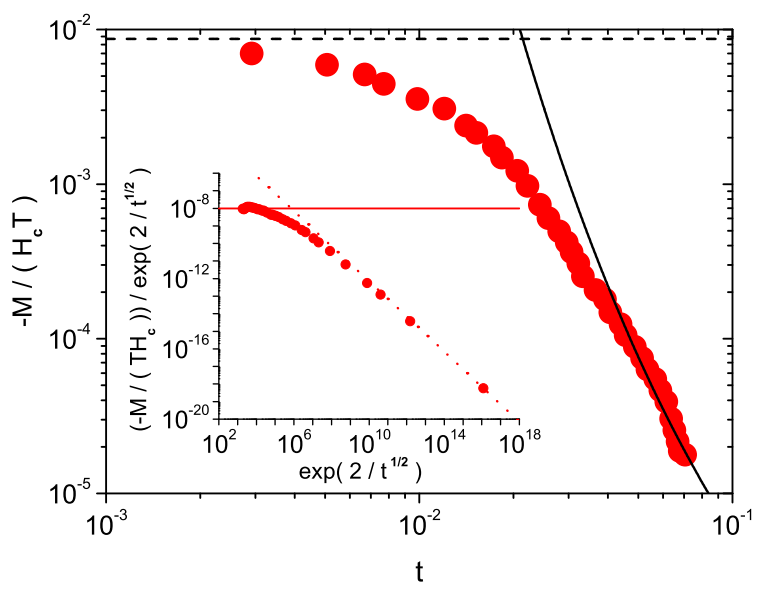

FIG. 4: $-M /\left(T H_{c}\right)$ vs. $t=T / T_{c}-1$ for $\mathrm{Bi}_{2} \mathrm{Sr}_{2} \mathrm{CaCu}_{2} \mathrm{O}_{8+\delta}$ at $H_{c}=10$ Oe with $T_{c}=84 \mathrm{~K}$ derived from the magnetization data of $\mathrm{Li}$ et al. [10]. The solid line is $-M /\left(T H_{c}\right)=10^{-8} \exp \left(2 / t^{1 / 2}\right)$ corresponding to Eq. (8) with $\xi_{a b}^{+} \propto \exp \left(b t^{-1 / 2}\right)$ with $b=1$ and the dashed one $-M /\left(T H_{c}\right)=8.710^{-3}$. The inset shows the finitesize scaling function in terms of $\left(-M /\left(T H_{c}\right)\right) / \exp \left(2 b t^{-1 / 2}\right)$ vs. $\exp \left(2 b t^{-1 / 2}\right)$ with $b=1$. The dotted line is $\left(-M /\left(T H_{c}\right)\right) / \exp \left(2 b t^{-1 / 2}\right)=0.007 / \exp \left(2 b t^{-1 / 2}\right)$, indicating the limiting magnetic field induced finite-size behavior and the solid one is $\left(-M /\left(T H_{c}\right)\right) / \exp \left(2 b t^{-1 / 2}\right)=10^{-8}$ marking the BKT limit.

the reduced temperature $t=T / T_{c}-1$, namely $\xi_{a b}^{+}=$ $a \exp \left(b t^{-1 / 2}\right)[2]$, with $b \approx 1$ a non-universal constant and $a$ related to the vortex core diameter. However, as $t$ decreases and with that $\xi_{a b}^{+}$approaches the limiting length $L_{H_{c}}=\sqrt{\Phi_{0} /\left(a H_{c}\right)}$ a finite size effect sets in and $\xi_{a b}^{+}$saturates in the limit $L_{H_{c}}<<\xi_{a b}^{+}$to $\xi_{a b}^{+}=L_{H_{c}}$. To describe the resulting crossover we introduce the finite-size scaling function $S$ [32] in terms of $\left(\widetilde{\xi_{a b}^{+}}\right)^{2}=$ $\left(\xi_{a b}^{+}\right)^{2} S\left(H_{c}\left(\xi_{a b}^{+}\right)^{2} / \Phi_{0}\right)=\left(\xi_{a b}^{+}\right)^{2} S\left(\left(\xi_{a b}^{+}\right)^{2} /\left(a L_{H_{c}}^{2}\right)\right)$ where $S\left(\left(\xi_{a b}^{+}\right)^{2} /\left(a L_{H_{c}}^{2}\right)\right) \rightarrow 1$ for $L_{H_{c}}>>\xi_{a b}^{+}$, $S\left(\left(\xi_{a b}^{+}\right)^{2} /\left(a L_{H_{c}}^{2}\right)\right) \rightarrow s a L_{H_{c}}^{2} /\left(\xi_{a b}^{+}\right)^{2}$ for $L_{H_{c}}<<\xi_{a b}^{+}$, and substitute $\widetilde{\xi_{a b}^{+}}$in Eq. (8) instead of $\xi_{a b}^{+}$. In Fig. 4 we depicted $-M /\left(T H_{c}\right)$ vs. $t=T / T_{c}-1$ at $H_{c}=10$ Oe and the inset shows the finite-size scaling function in terms of $\left(-M /\left(T H_{c}\right)\right) / \exp \left(2 b t^{-1 / 2}\right)$ vs. $\exp \left(2 b t^{-1 / 2}\right)$. It is seen that by approaching the transition temperature $-M /\left(T H_{c}\right)$ increases but saturates to $-M /\left(T_{c} H_{c}\right)=$ $8.710^{-3}$, in agreement with the behavior shown in Fig. 3. Sufficiently away from $T_{c}$ a crossover to the BKT behavior, requiring $L_{H_{c}}>>\xi_{a b}^{+}$and indicated by the solid line, can be anticipated. As the crossover extends over a rather extended temperature regime agreement with the leading BKT behavior is limited and the 3D- 
xy critical regime is not accessible. Nevertheless, the finite-size scaling function reveals the flow to BKT behavior is attained. To provide a consistency test we use $M(T=84 K) / H_{c}=6.6 / H_{c}$ (Fig. 3) and $d_{s}=15 \AA$ to estimate $a$ from $\left(-M /\left(T H_{c}\right)\right) / \exp \left(2 b t^{-1 / 2}\right)=10^{-8}$ (4) with the aid of Eqs. (77) and (8). The result is $a \simeq 9.2$ $\AA$, in reasonable agreement with the estimate of $\mathrm{Li}$ et al. [10].

We have seen that the conflicting observations in the highly anisotropic $\mathrm{Bi}_{2} \mathrm{Sr}_{2} \mathrm{CaCu}_{2} \mathrm{O}_{8+\delta}$, evidence for $\mathrm{BKT}$ behavior emerging from the magnetization data of $\mathrm{Li}$ et al. [10] and smeared 3D-xy behavior, stemming form the temperature dependence of the magnetic in-plane penetration depth [13, 16, 22] are a consequence of the rather small ratio, $\xi_{c}^{+} / \xi_{c}^{-}=\xi_{c 0}^{+} / \xi_{c 0}^{-} \simeq 0.45$, between the $c$-axis correlation length probed above $\left(\xi_{c}^{+}\right)$and below $\left(\xi_{c}^{-}\right)$ $T_{c}$ and the comparatively large anisotropy. The latter leads to critical amplitudes $\xi_{c 0}^{ \pm}$which are substantially smaller than the distance between two $\mathrm{CuO}_{2}$ double layers. In combination with $\xi_{c}^{+} / \xi_{c}^{-} \simeq 0.45$ and in contrast to the situation below $T_{c}$ the c-axis correlation length $\xi_{c}^{+}$exceeds the distance between two $\mathrm{CuO}_{2}$ double layers very close to $T_{c}$ only. Below this narrow temperature regime where 3D-xy fluctuations dominate, there is then an extended temperature regime left where the units with two $\mathrm{CuO}_{2}$ double layers are nearly uncoupled so that $2 \mathrm{D}$ thermal fluctuations dominate and BKT features are observable. As this behavior relies on the universal ratio $\xi_{c}^{+} / \xi_{c}^{-} \simeq 0.45$ and pronounced anisotropy it is not an artefact of $\mathrm{Bi}_{2} \mathrm{Sr}_{2} \mathrm{CaCu}_{2} \mathrm{O}_{8+\delta}$, but a generic feature of sufficiently anisotropic cuprate superconductors. Examples include underdoped $\mathrm{La}_{2-x} \mathrm{Sr}_{x} \mathrm{CuO}_{4}$ and $\mathrm{YBa}_{2} \mathrm{Cu}_{3} \mathrm{O}_{7-\delta}$ where the temperature dependence of the in-plane superfluid density does not reveal any trace of the universal jump [13, 14, 15, 16, 17, 18, 19, 20, 21].

[1] V.L. Berezinskii, Zh. Eksp. Teor. Fiz. 61, 1144 (1971); [Sov. Phys. JETP 34, 610 (1972)].

[2] J. M. Kosterlitz and D. J. Thouless, J. Phys. C 6, 1181 (1973); J. M. Kosterlitz, J. Phys. C 7, 1046 (1974).

[3] D. R. Nelson and J. M. Kosterlitz, Phys. Rev. Lett. 39, 1201 (1977).

[4] P. Minnhagen, Rev. Mod. Phys. 59, 1001 (1987).

[5] T. Schneider and J. M. Singer in Phase Transition Approach to High Temperature Superconductivity (Imperial College Press, London, 2000).
[6] T. Schneider, Physica B 326, 289 (2003).

[7] S. Vadlamannati, Q. Li, T. Venkatesan, W. L. McLean, and P. Lindenfeld, Phys. Rev. B 44, 7094 (1991).

[8] J. Corson et al., Nature (London) 398, 221 (1999).

[9] Yayu Wang, Z. A. Xu, T. Kakeshita, S. Uchida, S. Ono, Yoichi Ando, and N. P. Ong, Phys. Rev. B 64, 224519 (2001).

[10] Lu Li, Yayu Wang, M. J. Naughton, S. Ono, Yoichi Ando, and N. P. Ong, Europhys. Lett. 72, 451 (2005).

[11] Yayu Wang, Lu Li, M. J. Naughton, G. D. Gu, S. Uchida, and N. P. Ong, Phys. Rev. Lett. 95, 247002 (2005).

[12] D. Matthey, N. Reyren, and J.-M. Triscone, and T. Schneider, Phys. Rev. Lett. 98, 057002 (2007).

[13] T. Jacobs, S. Sridhar, Q. Li, G. D. Gu, and N. Koshizuka, Phys. Rev. Lett. 75, 4516 (1995).

[14] C. Panagopoulos, J. R. Cooper, and T. Xiang, Phys. Rev. B 57, 13422 (1998).

[15] C. Panagopoulos et al., Phys. Rev. B 60, 14617 (1999).

[16] K. D. Osborn, D. J. Van Harlingen, Vivek Aji, N. Goldenfeld, S. Oh, and J. N. Eckstein, Phys. Rev. B 68, 144516 (2003).

[17] A. Hosseini, et al., Phys. Rev. Lett. 93, 107003 (2004).

[18] D. M. Broun, et al., cond-mat/0509223

[19] R. Liang, D. A. Bonn, W. N. Hardy, and D. Broun, Phys. Rev. Lett. 94, 117001 (2005).

[20] Y. Zuev et al., Phys. Rev. Lett. 95,137002 (2005).

[21] A. Rüfenacht, J.-P. Locquet, J. Fompeyrine, D. Caimi, and P. Martinoli, Phys. Rev. Lett. 96, 227002 (2006).

[22] T. Schneider and D. Di Castro, Phys. Rev. B 69, 024502 (2004).

[23] V. G. Kogan, cond-mat/0611187

[24] S. Kawamata, K. Inoue, K. Okuda, and T. Sasaki, Physica B 246-247, 437 (1998).

[25] T. Watanabe, T. Fujii, and A. Matsuda1, Phys. Rev. Lett. 79, 2113 (1997).

[26] T. Schneider, in: The Physics of Superconductors, edited by K. Bennemann and J. B. Ketterson (Springer, Berlin, (2004)).

[27] A. Pelisetto and E. Vicari, Physics Reports 368, 549 (2002).

[28] T. Schneider, J. Hofer, M. Willemin, J.M. Singer, and H. Keller, Eur. Phys. J. B 3, 413 (1998).

[29] J. Hofer, T. Schneider, J. M. Singer, M. Willemin, H. Keller, C. Rossel, and J. Karpinski, Pys. Rev. B 60, 1332 (1999).

[30] J. Hofer, T. Schneider, J. M. Singer, M. Willemin, H. Keller, T. Sasagawa, K. Kishio, K. Conder, and J. Karpinski, Rev. B 62, 631 (2000).

[31] T. Schneider, Journal of Superconductivity, 17, 41 (2004).

[32] Finite-Size Scaling, edited by J. L. Cardy (North Holland, Amsterdam, 1988). 